Kiosov A. M. Effect of the botulinum toxin injections and endoscopic pneumocardiodilation on symptoms severity and treatment outcomes among patients with primary esophageal sphincter achalasia. Journal of Education, Health and Sport. 2021;11(10):252262. eISSN 2391-8306. DOI http://dx.doi.org/10.12775/JEHS.2021.11.10.023

https://apcz.umk.pl/JEHS/article/view/JEHS.2021.11.10.023

https://zenodo.org/record/5637091

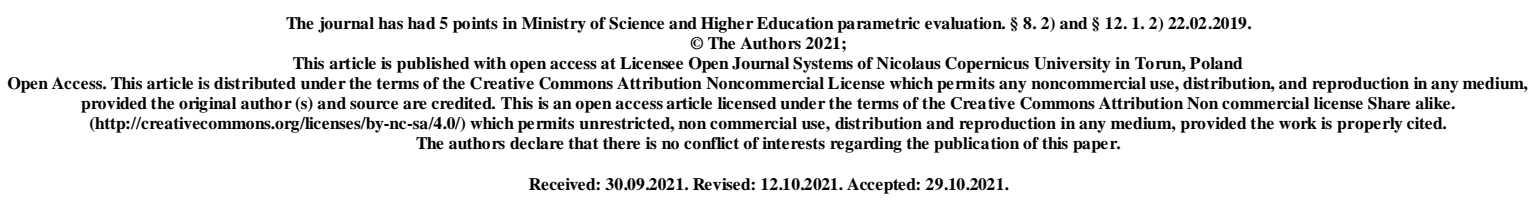

UDC: 616.329-008.22-036: 617-089.844

\title{
EFFECT OF THE BOTULINUM TOXIN INJECTIONS AND ENDOSCOPIC PNEUMOCARDIODILATION ON SYMPTOMS SEVERITY AND TREATMENT OUTCOMES AMONG PATIENTS WITH PRIMARY ESOPHAGEAL SPHINCTER ACHALASIA
}

\section{A. M. Kiosov}

\section{Zaporozhye State Medical University "University Clinic",}

Consultative Medical and Diagnostic Center, Department of Minimally Invasive Surgical and Endoscopic Interventions 83, Akademika Amosova St., 69600, Zaporizhzhia, Ukraine

$\mathrm{PhD}$, Associate Professor

ORCID 0000-0002-0212-1549

\section{Abstract}

Achalasia is a disorder of esophageal motility, which is manifested by symptoms of dysphagia, belching undigested food, respiratory symptoms (night cough, recurrent aspiration and pneumonia), chest pain and weight loss.

Aim of the study. To determine the possibilities and clinical effectiveness of combined approaches in the treatment of cardiac achalasia using endoscopic pneumocardiodilation and injections of botulinum toxin type A "Dysport" at various stages of the disease. 
Materials and methods. Injections of the drug botulinum toxin type A "Dysport" and balloon endoscopic pneumocardiodilation (EPCD) were used. The contents of the vial with botulinum toxin were diluted in $0.9 \%$ sodium chloride solution to obtain a solution containing $60 \mathrm{UA}$ in $1 \mathrm{ml}$. In each of the four quadrants of the LES, 2 injections of $0.5 \mathrm{ml}$ of the drug "Dysport" were made with a concentration of a solution of $30 \mathrm{UA}$ in $0.5 \mathrm{ml}$ at a distance of 1 $\mathrm{cm}$ from one another. The total dose was 250 units. The technique of isolated EPCD, was carried out with a Witzel balloon dilator, $40 \mathrm{~mm}$ in diameter, it required a thorough preoperative examination of the patient, including an assessment of the general condition, the presence and severity of body weight deficiency, water-electrolyte, protein, achlorhydric manifestations of the disease, as well as the propulsive ability of the esophagus through X-ray examination.

Results. The combined endoscopic method significantly improves the results of treatment of esophageal cardiac achalasia, having a direct impact on the pathogenetic mechanisms of its development. Given the effectiveness of treatment of esophageal cardiac achalasia by botulinum toxin injection and pneumocardiodilation, this method can be used in patients with comorbid pathology and high surgical risk as an alternative to laparoscopic Heller procedure (cardiomyotomy), especially in older patients. Unsatisfactory results of the combined endoscopic method of treatment of stage III-IV cardiac achalasia for up to 2 months are an indication for surgical treatment of this pathology.

Conclusions. Given the significant increase in knowledge about this nosology in recent years, there is a need for a comprehensive, evidence-based study of new promising treatments for patients, covering all aspects of the disease.

Key words: esophageal achalasia; endoscopic pneumocardiodilation; injections of botulinum toxin type A "Dysport".

Revalence. Achalasia is a disorder of esophageal motility, which is manifested by symptoms of dysphagia, belching undigested food, respiratory symptoms (night cough, recurrent aspiration and pneumonia), chest pain and weight loss. This esophageal motor disorder is characterized by aperistalsis of the esophageal body and lack of relaxation of the lower sphincter in response to swallowing. It affects people of both sexes and all ages [1]. Since its first mention in 1674 by Thomas Willis, spasm or inability to relax the lower esophageal sphincter (LES) has been identified as the cause of achalasia, leading to disruption of the flow of food into the stomach and subsequent stagnation of food and secretions in the esophagus [2]. 
Achalasia can be primary (idiopathic) or secondary. At secondary achalasia the reason of degeneration of nerve fibers of a gullet is known. Pathophysiologically, achalasia is caused by the loss of inhibitory ganglion cells in the myenteric plexus, which coordinate esophageal peristalsis and LES relaxation. Since its initial description, several studies have attempted to examine the causes that can cause disease, such as viral infection, other environmental factors, autoimmunity, and genetic factors. However, the exact pathogenesis of primary achalasia is still unknown [3].

Idiopathic achalasia is rare, the average birth rate in adults is $0.3-1.63$ per 100,000 people per year and 0.18 per 100,000 people per year among children under 16 years [4]. In adults, achalasia occurs with equal frequency in men and women, regardless of race, the incidence increases with age. In most studies, the average age of diagnosis was over 50 years [5]. The average incidence among people over 80 years is 17 per 100,000 people per year [6]. The challenges of treating patients with achalasia can be considered in terms of reducing symptoms and, consequently, improving quality of life. Because evidence of the use of standardized questionnaires in clinical settings is limited, careful clinical evaluation of symptoms before and after therapy should be used to assess treatment success [7]. Treatment can prevent the disease from progressing to the terminal stage and the appearance of late complications, such as aspiration and carcinogenesis.

Despite treatment, some patients will experience persistent or recurrent symptoms that significantly impair quality of life. In some cases, treatment does not lead to significant improvement at all (persistent symptoms) [8]. In other cases, the period of initial improvement is followed by further recurrence. In general, the first option assumes that the initial treatment was incomplete, while the second option can be caused by a number of reasons. There is no universal definition of what constitutes constancy or recurrence of symptoms. In most studies, a score on the Eckard scale above 3 or a reduction in symptoms of less than $50 \%$ is considered treatment ineffective [9].

Given the significant increase in knowledge about this nosology in recent years, there is a need for a comprehensive, evidence-based study of new promising treatments for patients, covering all aspects of the disease.

Aim of the study. To determine the possibilities and clinical effectiveness of combined approaches in the treatment of cardiac achalasia using endoscopic pneumocardiodilation and injections of botulinum toxin type A "Dysport" at various stages of the disease. 
Materials and methods. Injections of the drug botulinum toxin type A "Dysport" and balloon endoscopic pneumocardiodilation (EPCD) were used. The contents of the vial with botulinum toxin were diluted in $0.9 \%$ sodium chloride solution to obtain a solution containing $60 \mathrm{UA}$ in $1 \mathrm{ml}$. In each of the four quadrants of the LES, 2 injections of $0.5 \mathrm{ml}$ of the drug "Dysport" were made with a concentration of a solution of $30 \mathrm{UA}$ in $0.5 \mathrm{ml}$ at a distance of 1 $\mathrm{cm}$ from one another. The total dose was 250 units. The technique of isolated EPCD, was carried out with a Witzel balloon dilator, $40 \mathrm{~mm}$ in diameter, it required a thorough preoperative examination of the patient, including an assessment of the general condition, the presence and severity of body weight deficiency, water-electrolyte, protein, achlorhydric manifestations of the disease, as well as the propulsive ability of the esophagus through X-ray examination. The main parameters of the $\mathrm{X}$-ray examination were the following: the time and type of the evacuation, the preoperative and postoperative assessment of the propulsive capacity of the LES with an assessment of the degree of delay of the contrast mass. The requirement for maximum safety for patients was met by a phased expansion of the cardia, first with a balloon with a diameter of $30 \mathrm{~mm}$ at a pressure of $180-200 \mathrm{~mm} \mathrm{Hg}$ with a gradual increase in the diameter of the balloon to $40-50 \mathrm{~mm}$ and the pressure in it to $340-360 \mathrm{~mm} \mathrm{Hg}$, the duration of EPCD ranged from 2 up to 6 minutes and frequency (in ascending diameter and pressure in the cylinder) from 5 to 10 times. The combined technique of EPCD and botulinum toxin injection "Dysport" was carried out using a two-channel endoscope FUJINON EG 530D and an endoscopic video system EPX $4450 \mathrm{HD}$ in the clinic of the Department of Minimally Invasive Surgical and Endoscopic Interventions and the Department of Faculty Surgery of Zaporozhye State Medical University. Under visual control, through the first channel of the endoscope with a diameter of $3.2 \mathrm{~mm}$, a Micro-Tech pneumatic balloon with a diameter of $18 \mathrm{~mm}$ and $20 \mathrm{~mm}$ was passed to the cardiac sphincter; and the balloon was held unfolded. The duration of the combined endoscopic method ranged from 8 to 12 minutes.

Results. Clinical case No. 1. Patient I. was born in 1974, came to the clinic with complaints of dysphagia, a feeling of a "lump" behind the breastbone after eating and the need to drink water after food, sometimes take a forced position, raising her arms up or perform moderate jumping with raised arms, as if pushing food into the stomach. These manifestations have been observed for 2 years. During the last 5-6 months, there has been a significant increase in pain and the frequency of the above symptoms. Preoperative radiography of the esophagus with barium: the act of swallowing is not disturbed, the barium suspension fills the esophagus, there is a slight antiperistalsis with an initial evacuation of barium from the 
esophagus into the stomach at 2 minutes, intraesophageal retention of the contrast mass for more than 7 minutes. The symptom of the "mouse tail" is determined. Endoscopic characteristics: the esophagus is freely passable, the mucous membrane is unremarkable, the cardiac sphincter is closed, cyanotic, passable with difficulty. In retroversion, the cardiac fold tightly encloses and, as it were, "closes" the endoscope. Diagnosed with stage II cardiac achalasia. The combined endoscopic treatment was carried out: injection of the drug "Dysport" into four points of the cardiac sphincter and isolated EPCD with a frequency of pressurization in the balloon up to 4 times and an exposure of 1 to 3 minutes.

A control radiography with barium on the 7th day after endoscopic treatment was carried out: the act of swallowing was not disturbed, barium completely and evenly fills the lumen of the esophagus, there is a slight antiperistalsis, after which barium is completely evacuated into the stomach. In this case, a single carrying out of the combined endoscopic method using the drug "Dysport" determined the positive dynamics and a significant decrease in the clinical manifestations of cardiac achalasia within 6 months with no relapses for 8-9 months.

Clinical case No. 2. Patient V. was born in 1961, came to the clinic with complaints of chest pains of a spastic nature, feeling of a "lump" and not passing food, arising after eating, partially relieved by taking nitroglycerin. A preoperative radiography of the esophagus with barium was performed: the act of swallowing was not disturbed, the barium suspension fills the esophagus, antiperistaltic waves with the phenomenon of hypertonicity of the esophageal wall are determined, the cardiac sphincter is closed, the symptom of "mouse tail" is determined, the gas bubble of the stomach is not detected. The initial evacuation of barium was slowed down to 10 minutes, the full evacuation with the remaining part of the contrast was extended to 30 minutes. The diagnosis was established: achalasia of the cardiac sphincter II-III stage. The patient underwent an isolated EPCD with a Vitzel balloon. Preliminary dilatation of the cardia was performed twice with an exposure of 2 minutes and a pressure inside the balloon up to $200 \mathrm{~mm} \mathrm{Hg}$ with a balloon dilator with a diameter of $20 \mathrm{~mm}$, after which a Witzel balloon dilator with a diameter of $40 \mathrm{~mm}$ was inserted. There were made two cardiomyodilations with an exposure of 3 minutes and a pressure of up to $220 \mathrm{~mm} \mathrm{Hg}$. At the control examination in the area of the cardia, single, submucous hemorrhages are determined, which indicated the adequacy of the procedure.

Control radiography of the esophagus with barium was performed on the 7th day after the manipulation: a third part of the contrast is in the esophagus, the cardia is open, gaping, evacuation is slightly slowed down, portioned, almost complete after inspection after 10 
minutes and the patient's polypositional location. Against the background of treatment, the patient notes an improvement in well-being, chest pains disappeared, food passes freely. There are episodes of functional dyspepsia in the form of heartburn, rumination and reflux, of an irregular nature, usually caused by overeating or an error in the diet. Discussing the presented clinical case, it can be noted that the so-called therapeutic regression of the disease takes place. This example reflects the need for a deeper endomorphological separation of the II and III stages, and in some cases, detailing the I and IV stages of the disease with its complications.

Clinical case No. 3. Patient N. was born in 1943, came to the clinic with complaints of the occurrence of chest pain, feeling of a "lump", stuck and delayed food in the esophagus with the first portion of food, the patient takes a forced position (raises the upper limbs and performs a deflection in an upright position, the duration of the exercise is up to 15-20 seconds with a frequency of 3 to 15 times), in rare cases, performs retraction movements of the anterior abdominal wall up to 30-40 times with an insignificant clinical effect. Radiography of the esophagus with barium: the act of swallowing occurs in small portions, is somewhat difficult, the esophagus, when filled with barium, has a clear wavy, contour, "dumbbell" shape with alternating areas of expansion and narrowing of the lumen, up to 25 $27 \mathrm{~cm}$ in length. After the contrast is washed from the esophagus, a narrowing is observed the supraphrenic segment up to 5-6 cm long, the gas bubble of the stomach is small. The diagnosis was established: achalasia of the cardia of III-IV stages, severe motor-evacuation disorders of the esophagus, the threat of spontaneous perforation. Diagnostic fibrogastroduodenoscopy was performed: the esophagus is freely passable, there are areas of narrowing at the level of the middle and lower parts, the mucous membrane of the esophagus in the areas of expansion is pale, thinned, there are areas of mucous erosion and inflammation, places of fibrin overlap, pronounced vitreous edema of the esophageal mucosa is determined, in places - stasic changes. The lumen of the esophagus is unevenly curved, peristalsis is reduced or absent at the level of the middle third with a spastic component in the area of the muscle ring $\mathrm{A}$, the apparatus passes through the muscle ring with difficulty. The cardiac sphincter is spasmodic, hypertonic, the pattern of palisade vessels is preserved fragmentarily. In retroversion, the cardiac fold does not completely cover the endoscope, and the epithelium of the esophagus prolapses. Endoscopic treatment of esophageal achalasia was carried out using the combined method of the injection of the drug "Dysport" and the simultaneous placement of a balloon for dilatation with a diameter of $18 \mathrm{~mm}$. 
Within 2 months, the patient underwent three sessions of combined endoscopic treatment, but no clear positive effect was observed, the clinical picture of the disease remained, confirmed by the data of endoscopic and X-ray studies. For further treatment, the patient was referred to the surgical department.

Discussion. Pharmacological treatment of achalasia with botulinum toxin type A, a neurotoxin that blocks the release of acetylcholine from nerve endings, is widely used. It is administered at a dose of 80-100 units in four or eight quadrants directly into the LES through a needle for sclerotherapy during endoscopy of the upper gastrointestinal tract [10]. Botulinum toxin is a safe and effective treatment with few side effects. In more than $80 \%$ of cases, the clinical response occurs after 1 month, but the response disappears rapidly, and less than $60 \%$ of patients experience remission after 1 year. The results of five randomized trials comparing botulinum toxin with EPCD and botulinum toxin with laparoscopic myotomy initially showed relief from dysphagia, but rapid deterioration in patients receiving botulinum toxin after 6-12 months [11]. Therefore, botulinum toxin, as with nitrates and calcium channel blockers, should only be used as an intermediate before longer treatment or in high-risk patients.

There is no convincing evidence that treatment with smooth muscle relaxants (calcium blockers, phosphodiesterase inhibitors or nitrates) provides relief of symptoms in adults with achalasia. None of the studies were of high enough quality, did not have a sufficient sample size and adequate endpoints to answer this question. Smooth muscle relaxant treatment can cause side effects, so it is sometimes not recommended. This treatment option, of course, should not delay more effective endoscopic or surgical treatment [12].

Endoscopic injection of botulinum toxin in the LES was compared with laparoscopic Heller myotomy (LHM) or EPCD in several studies. All the results of these studies point to the same conclusion - injections reduce LES pressure, reduce signs of stagnation and symptoms in the short term, but usually the symptoms and signs of the disease recur over time. EPCD treatments and botulinum toxin injection are equally effective in the short term, while PD is a more effective endoscopic treatment in the long run (over six months). LHM treatment and botulinum toxin injection are equally effective in the short term; LHM is a more effective treatment in the long run (more than six months) [13].

EPCD, in which the LES is ruptured by forced stretching using air-filled balloons, has been simplified using the Rigiflex microinvasive balloon system (Boston Scientific, Marlborough, Massachusetts, USA). These polyethylene cylinders are available in three diameters $(30,35$ and $40 \mathrm{~mm})$, are mounted on a flexible catheter, which is placed over the 
wire conductor during endoscopy. The actual protocol varies depending on the center. Under radioscopic control, the balloon is placed across the LES and gradually inflated until the waist becomes flat. The most popular method is a gradual dilatation protocol, starting with a $30 \mathrm{~mm}$ cylinder. Subsequent dilatations are divided into 2-4-week intervals based on the relief of symptoms associated with repeated measurements of LES pressure or improved esophageal emptying. EPCD is usually performed in an outpatient setting; the patients are observed for 26 hours, and they can return to normal activities the next day [14].

In a review of more than 1,100 patients (24 studies) with a mean follow-up of 37 months, EPCD Rigiflex resulted in symptom relief from good to excellent in $74 \%, 86 \%$, and $90 \%$ of patients receiving 30, 35, and $40 \mathrm{~mm}$ diameter cylinders, respectively [154 ]. Within 4-6 years, almost a third of patients have recurrences of symptoms; however, long-term remission can be achieved in almost all of these patients by re-dilation using an on-demand strategy based on recurrence of symptoms. Patients with the best results after EPCD are patients older than 40 years, women and patients with type II achalasia. The most costeffective method of treating achalasia for 5-10 years after the procedure is EPCD according to the findings of this study [15].

Contraindications for EPCD are poor cardiopulmonary status or other comorbidities that may interfere with surgery in case of esophageal perforation. EPCD can be safely performed after a failed LHM, although larger diameter cylinders are often required. Up to $33 \%$ of patients have procedure-related complications after EPCD, but most are minor, including chest pain, aspiration pneumonia, bleeding, transient fever, rupture of the mucosa without perforation, and esophageal hematoma [16]. Esophageal perforation is the most serious complication, with an overall incidence of $2.0 \%$ (range $0-16 \%$ ) in experienced endoscopists, of whom 50\% required surgery. However, in a recent series of 16 transmural perforations, all cases were treated conservatively. Small perforations and painful deep tears can be treated with antibiotics and complete parenteral nutrition for days or weeks. However, surgical treatment by thoracotomy is best for large perforations with significant mediastinal contamination. Most perforations occur during the initial dilatation; difficulty keeping the balloon in place is a potential risk factor. Although no other predictors of perforation were found, a European achalasia study reported more perforations, especially in elderly patients when the first EPCD was made with a $35 \mathrm{~mm}$ balloon compared to a $30 \mathrm{~mm}$ balloon. Complications of severe gastroesophageal reflux disease after EPCD are rare, but $15-35 \%$ of patients develop heartburn, which is improved by proton pump inhibitors [17]. 
EPCD also was compared with endoscopic injections of botulinum toxin, per-oral endoscopic myotomy (POEM) and LHM. An important factor in comparing different studies is the EPCD regime, which varies widely. Generally speaking, multiple-dose treatment regimens performed in case of recurrent symptoms increase efficacy. One series of EPCD is less effective than LHM or POEM, and there is no difference in safety between the two treatment groups. In studies that allowed re-dilation in recurrence of symptoms, the effectiveness of EPCD was usually close to the effectiveness of LHM with a similar safety profile. Due to the risk of perforation, it is always recommended to start with a $30 \mathrm{~mm}$ balloon in patients with achalasia without treatment. The second dilatation of $35 \mathrm{~mm}$ will extend the time to relapse [18].

Limitations in research. Financial resources, method and data collection.

Prospects for further research. Prospects for further research are to continue further investigation of clinical effectiveness of combined approaches in the treatment of cardiac achalasia at various stages of the disease and in different terms.

Conclusions. 1. The combined endoscopic method significantly improves the results of treatment of esophageal cardiac achalasia, having a direct impact on the pathogenetic mechanisms of its development.

2. Given the effectiveness of treatment of esophageal cardiac achalasia by botulinum toxin injection and pneumocardiodilation, this method can be used in patients with comorbid pathology and high surgical risk as an alternative to laparoscopic Heller procedure (cardiomyotomy), especially in older patients.

3. Unsatisfactory results of the combined endoscopic method of treatment of stage IIIIV cardiac achalasia for up to 2 months are an indication for surgical treatment of this pathology.

Conflicts of interest. Neither author has actual or potential conflicts of interest.

\section{References}

1. Oude Nijhuis RAB, Zaninotto G, Roman S, et al. European guidelines on achalasia: United European Gastroenterology and European Society of Neurogastroenterology and Motility recommendations. United European Gastroenterol J. 2020;8(1):13-33. doi:10.1177/2050640620903213

2. Richter JE. Achalasia - an update. J Neurogastroenterol Motil. 2010;16(3):232242. doi:10.5056/jnm.2010.16.3.232 
3. Ghoshal UC, Daschakraborty SB, Singh R. Pathogenesis of achalasia cardia. World J Gastroenterol. 2012;18(24):3050-3057. doi:10.3748/wjg.v18.i24.3050

4. Schlottmann F, Patti MG. Esophageal achalasia: current diagnosis and treatment. Expert Rev Gastroenterol Hepatol. 2018 Jul;12(7):711-721. doi: 10.1080/17474124.2018.1481748. Epub 2018 Jun 8. PMID: 29804476.

5. Vaezi MF, Pandolfino JE, Yadlapati RH, Greer KB, Kavitt RT. ACG Clinical Guidelines: Diagnosis and Management of Achalasia. Am J Gastroenterol. 2020 Sep;115(9):1393-1411. doi: 10.14309/ajg.0000000000000731. PMID: 32773454.

6. Schlottmann F, Patti MG. Primary Esophageal Motility Disorders: Beyond Achalasia. Int J Mol Sci. 2017 Jun 30;18(7):1399. doi: 10.3390/ijms18071399. PMID: 28665309; PMCID: PMC5535892.

7. Khashab MA, Vela MF, Thosani N, Agrawal D, Buxbaum JL, Abbas Fehmi SM, Fishman DS, Gurudu SR, Jamil LH, Jue TL, Kannadath BS, Law JK, Lee JK, Naveed M, Qumseya BJ, Sawhney MS, Yang J, Wani S. ASGE guideline on the management of achalasia. Gastrointest Endosc. 2020 Feb;91(2):213-227.e6. doi: 10.1016/j.gie.2019.04.231. Epub 2019 Dec 13. PMID: 31839408.

8. Gasanov AM, Aliev NA, Danielyan SN. Akhalaziia kardii [Esophageal achalasia]. Khirurgiia (Mosk). 2019;(2):72-77. Russian. doi: 10.17116/hirurgia201902172. PMID: 30855594.

9. Huang L, Pimentel M, Rezaie A. Do Jackhammer contractions lead to achalasia? A longitudinal study. Neurogastroenterol Motil. 2017 Mar;29(3). doi: 10.1111/nmo.12953. Epub 2016 Sep 23. PMID: 27660053.

10. Letranchant A, Pigneur B, Flament M, Godart N. Eating disorder or oesophageal achalasia during adolescence: diagnostic difficulties. Eat Weight Disord. 2020 Feb;25(1):87-90. doi: 10.1007/s40519-018-0513-2. Epub 2018 May 17. PMID: 29774515.

11. Wagner A, Ravi K. Achalasia Caused by a Peptic Stricture. Clin Gastroenterol Hepatol. 2017 Apr;15(4):A19-A20. doi: 10.1016/j.cgh.2016.12.005. Epub 2016 Dec 15. PMID: 27988381.

12. Fisichella PM, Orthopoulos G, Holmstrom A, Patti MG. The surgical management of achalasia in the morbid obese patient. J Gastrointest Surg. 2015 Jun;19(6):1139-43. doi: 10.1007/s11605-015-2790-7. Epub 2015 Mar 11. PMID: 25759076.

13. Ross D, Richter J, Velanovich V. Health-related quality of life and physiological measurements in achalasia. Dis Esophagus. 2017 Feb 1;30(2):1-5. doi: 10.1111/dote.12494. PMID: 27629426. 
14. Patti MG, Fisichella PM. Controversies in management of achalasia. J Gastrointest Surg. 2014 Sep;18(9):1705-9. doi: 10.1007/s11605-014-2556-7. Epub 2014 Jun 28. PMID: 24972973.

15. Miranda LEC, Miranda ACG, Lima DL. An Unbelievable Esophagus. Gastroenterology. 2020 Oct;159(4):e11-e12. doi: 10.1053/j.gastro.2020.03.077. Epub 2020 Apr 18. PMID: 32311355.

16. Oude Nijhuis RAB, Bergman JJGHM, Takkenberg RB, Fockens P, Bredenoord AJ. Non-surgical treatment of esophageal perforation after pneumatic dilation for achalasia: a case series. Scand J Gastroenterol. 2020 Oct;55(10):1248-1252. doi: 10.1080/00365521.2020.1817541. Epub 2020 Sep 13. PMID: 32924655.

17. Andolfi C, Fisichella PM. Meta-analysis of clinical outcome after treatment for achalasia based on manometric subtypes. Br J Surg. 2019 Mar;106(4):332-341. doi: 10.1002/bjs.11049. Epub 2019 Jan 28. PMID: 30690706.

18. Kalantari M, Hollywood A, Lim R, Hashemi M. Mapping the experiences of people with achalasia from initial symptoms to long-term management. Health Expect. 2021 Feb;24(1):131-139. doi: 10.1111/hex.13160. Epub 2020 Nov 19. PMID: 33210821; PMCID: PMC7879551. 